\title{
Pseudospin Symmetry as a Bridge between Hadrons and Nuclei
}

\author{
Joseph N. Ginocchio \\ Theoretical Division, Los Alamos National Laboratory, Los Alamos 87505, NM, USA; gino@lanl.gov or \\ j.ginocchio@comcast.net; Tel.: +505-667-5630 \\ Academic Editor: Stefan Frauendorf \\ Received: 11 January 2016; Accepted: 23 February 2016; Published: 18 March 2016
}

\begin{abstract}
Atomic nuclei exhibit approximate pseudospin symmetry. We review the arguments that this symmetry is a relativistic symmetry. The condition for this symmetry is that the sum of the vector and scalar potentials in the Dirac Hamiltonian is a constant. We give the generators of pseudospin symmetry. We review some of the predictions that follow from the insight that pseudospin symmetry has relativistic origins. We show that approximate pseudospin symmetry in nuclei predicts approximate spin symmetry in anti-nucleon scattering from nuclei. Since QCD sum rules predict that the sum of the scalar and vector potentials is small, we discuss the quark origins of pseudospin symmetry in nuclei and spin symmetry in hadrons.
\end{abstract}

Keywords: pseuspin symmetry; quarks in nuclei; relatiistic symmetry in nuclei

\section{Introduction}

Quarks in hadrons are relativistic while for the most part the properties of nuclei can be explained by non-relativistic physics. However, a quasi-degeneracy in the single nucleon states of spherical nuclei has a simple explanation as a relativistic symmetry. The states in question have quantum numbers $\left(n \ell_{j}, n^{\prime} \ell_{j^{\prime}}^{\prime}\right)$ where $n^{\prime}=n-1, \ell^{\prime}=\ell+2, j^{\prime}=j+1$ and $n, \ell, j$ are the radial, orbital angular momentum, and total angular momentum quantum numbers, respectively [1,2]. These quasi-degeneracies persist in recent measurements in nuclei far from stability [3]. If the average of the orbital angular momenta is defined as a pseudo-orbital angular momentum $(\tilde{\ell})$ and if a pseudospin $\left(\tilde{s}=\frac{1}{2}\right)$ is coupled to the pseudo-orbital angular momentum, the total angular momenta will be $\left(j=\tilde{\ell} \pm \frac{1}{2}\right)$. For example, for the $\left(1 s_{\frac{1}{2}}, 0 d_{\frac{3}{2}}\right)$ orbits, $\tilde{\ell}=1$, which gives the total angular momenta $j=\frac{1}{2}, \frac{3}{2}$. Subsequently pseudospin doublets in deformed nuclei were discovered [4]. Pseudospin symmetry was later revealed to be a relativistic symmetry of the Dirac Hamiltonian [5,6]. Furthermore, QCD sum rules predict that the vector and scalar potentials of nucleons in nuclear matter approximately satisfy the conditions for pseudospin symmetry. Therefore, perhaps pseudospin symmetry can be a bridge to understanding nuclei in terms of quarks.

In this paper, we will review the symmetries of the Dirac Hamiltonian[5,7]. Data on hadrons suggest they have a spin symmetry. Spin symmetry is not usually thought of as a relativistic symmetry but we show in the next Section that the Dirac Hamiltonian does have a spin symmetry in a certain limit. Then we will show that the Dirac Hamiltonian has a pseudospin symmetry and we give a short review of the evidence of pseudospin symmetry in nuclei. We then discuss connections of pseudospin symmetry with QCD. 


\section{Symmetries of the Dirac Hamiltonian}

The Dirac Hamiltonian with a Lorentz scalar potential, $V_{S}(\vec{r})$, and a potential which is the fourth component of a Lorentz vector potential, $V_{V}(\vec{r})$, is

$$
H=\vec{\alpha} \cdot \vec{p}+\beta\left(V_{S}(\vec{r})+M\right)+V_{V}(\vec{r})
$$

where $\vec{\alpha}, \beta$ are the Dirac matrices, $\vec{p}$ is the momentum, $M$ is the mass, $\vec{r}$ is the radial coordinate, and the velocity of light is set equal to unity, $\mathrm{c}=1$.

\subsection{Spin Symmetry: A Symmetry of the Dirac Hamiltonian}

The Dirac Hamiltonian has spin symmetry when the difference of the vector and scalar potentials in the Dirac Hamiltonian is a constant, $V_{S}(\vec{r})-V_{V}(\vec{r})=C_{s}$ [8]. Hadrons [9] and anti-nucleons in a nuclear environment have spin symmetry [10]. These are relativistic systems and normally, in such systems, we would expect large spin-orbit splittings, but, in this limit, spin doublets are degenerate. The generators for this SU(2) spin algebra, $\vec{S}$, which commute with the Dirac Hamiltonian with any potential $V(\vec{r})$, spherical or deformed, $[H, \vec{S}]=0$, are given by [11]

$$
\vec{S}=\left(\begin{array}{cc}
\vec{s} & 0 \\
0 & U_{p} \vec{s} U_{p}
\end{array}\right)
$$

where $\vec{s}=\vec{\sigma} / 2$ are the usual spin generators, $\vec{\sigma}$ the Pauli matrices, and $U_{p}=\vec{\sigma} \cdot \hat{p}$ is the helicity unitary operator introduced in [12] and $\hat{p}=\frac{\vec{p}}{p}$, the unit momentum. The generators are four by four matrices as appropriate for the Dirac Hamiltonian.

\subsection{Pseudospin Symmetry: A Symmetry of the Dirac Hamiltonian}

Another SU(2) symmetry of the Dirac Hamiltonian occurs when the sum of the vector and scalar potentials in the Dirac Hamiltonian is a constant, $V_{S}(\vec{r})+V_{V}(\vec{r})=C_{p s}$ [8]. The generators for this SU(2) algebra $\overrightarrow{\widetilde{S}}$, which commute with the Dirac Hamiltonian with any potential $V(\vec{r})$, spherical or deformed, $[H, \overrightarrow{\tilde{S}}]=0$, are given by [11]

$$
\overrightarrow{\widetilde{S}}=\left(\begin{array}{cc}
U_{p} \vec{s} U_{p} & 0 \\
0 & \vec{s}
\end{array}\right)
$$

This symmetry was shown to be pseudospin symmetry [5]. The eigenfunctions of the Dirac Hamiltonian in this limit will have degenerate doublets of states, one of which has pseudospin aligned and the other with pseudospin unaligned. The "upper" matrix of the pseudospin generators in Equation (3), $U_{p} \vec{s} U_{p}$, have the spin intertwined with the momentum which enables the generators to connect the states in the doublet, which differ by two units of angular momentum. In finite nuclei both potentials go to zero at infinite distances. Hence $C_{p s}=0$ and the scalar potential will be equal in magnitude but opposite in sign to the vector potential in the pseudospin limit. The approximate equality in the magnitude of the vector and scalar fields in nuclei and their opposite sign have been confirmed in relativistic mean field theories [6] and in QCD sum rules [6,13].

\section{Consequences of Relativistic Pseudospin Symmetry}

One immediate consequence of pseudospin symmetry as a relativistic symmetry is that the "lower" matrix of the pseudospin generators in Equation (3), $\vec{s}$, does not change the radial wavefunction of the "lower" component of the Dirac eigenfunctions. Hence this symmetry predicts that the radial wavefunctions of the "lower" component are the same for the two states in the doublet. Previous to this discovery many relativistic mean field calculations of nuclear properties had been made. Hence this prediction was tested with existing calculations and, indeed, these wavefunctions are very similar for both spherical $[14,15]$ and deformed nuclei $[16,17]$. Because of the momentum dependence of 
the "upper" matrix of the generators the relationship between the "upper" components involves a differential equation and these have also been tested in spherical [18] and deformed nuclei [17] with success.

Magnetic dipole and Gamow-Teller transitions between the two single-nucleon states in pseudospin doublets are forbidden non-relativistically because the states differ by two units of angular momentum. However, they are not forbidden relativistically which means that they are proportional to the lower component of the Dirac eigenfunction. This leads to a condition between the magnetic moments of the states and the magnetic dipole transtion between them because the radial amplitudes of the lower components of the two states in a pseudospin doublet are equal. Therefore, the magnetic dipole transition between the two states in the doublet can be predicted if the magnetic moments of the states are known $[6,19]$. Likewise pseudospin symmetry also predicts Gamow-Teller transitions between a state in a parent nucleus to the partner state in the daughter nucleus if the Gamow-Teller transition to the same states in the parent and daughter nucleus is known. We do not have space to discuss these relationships in detail but one example occurs in the mirror nuclei ${ }_{19}^{39} \mathrm{~K}_{20}$ and ${ }_{20}^{39} \mathrm{Ca}_{19}$. The ground state and first excited state of ${ }_{19}^{39} \mathrm{~K}_{20}$ are interpreted as a $0 d_{3 / 2}$ and $1 s_{1 / 2}$ proton hole respectively, while the ground state and first excited state of ${ }_{20}^{39} \mathrm{Ca}_{19}$ are interpreted as a $0 d_{3 / 2}$ and $1 s_{1 / 2}$ neutron hole respectively. These states are members of the $\tilde{n}_{r}=1, \tilde{\ell}=1$ pseudospin doublet. Using the magnetic moment of ${ }^{39} \mathrm{Ca}$ a transition rate is calculated which is only about $37 \%$ larger than the measured value. However, the two states in the doublet are not pure single-particle states. A modification of these relations has been derived which take into account the fact that these states are not pure single particle states $[6,20]$. The modified relations give a transition rate that agrees with the measured value to within experimental error. Again using the mass 39 nuclei, the Gamow-Teller transitions from the ground state of ${ }^{39} \mathrm{Ca}$ to the ground and first excited state of ${ }^{39} \mathrm{~K}$ are known, which is enough information to predict the transition from the ground state to the excited state. In the non-relativistic shell model an effective tensor term $g_{e f f}\left[Y_{2} \sigma\right]^{(1)}$ is added to the magnetic dipole operator and the Gamow-Teller operator to produce a transition, where $g_{e f f}$ is a calculated effective coupling constant. However, the magnetic dipole transition calculated between the same states is an order of magnitude lower than the experimental transition [21] although the calculated Gamow-Teller agrees with the experimental value within the limits of experimental and theoretical uncertainty. This inconsistency has been a puzzle for the non-relativistic shell model. On the other hand the relativistic single-nucleon model gives a consistent description of both of these transitions. A global prediction of magnetic dipole transitions throughout the periodic table has had reasonable success as well $[6,20]$. However, a global prediction of Gamow-Teller transitions have not been done yet. Pseuodspin symmetry can also be used to relate quadrupole transitions between multiplets [6].

\section{Anti-nucleon in a Nuclear Environment}

Charge conjugation changes a nucleon into an anti-nucleon. Under charge conjugation the scalar potential of a nucleon remains invariant while the its vector potential changes sign. Hence the pseudospin condition $V_{S}(\vec{r})+V_{V}(\vec{r}) \approx C_{p s}$ becomes the spin condition $\bar{V}_{S}(\vec{r})-\bar{V}_{V}(\vec{r}) \approx C_{p s}=\bar{C}_{s}$ where $\bar{V}_{S, V}(\vec{r})$ are the scalar and vector potentials of an anti-nucleon in a nuclear environment respectively. Hence approximate pseudospin for nucleons predicts approximate spin symmetry for anti-nucleons in a nuclear environment. This conjecture is confirmed in relativistic mean field theory [22]. Since the potentials are also very deep we would expect approximate $U(3)$ symmetry as well [23].

Of course the anti-nucleons scattered from nuclei experience a strong annihilation potential while nucleons scattered from nuclei do not. However, since the nucleons do not feel an annihilation potential, this means that the sum of the scalar and vector annihilation potentials for nucleons must be zero which means that the difference of the anti-nucleon annihilation potentials is zero as well. Hence the anti-nucleon annihilation potentials conserve spin symmetry as well. 
The sparse data on the scattering of polarized anti-protons on nuclei supports the prediction that spin symmetry is approximately conserved [24]. Perhaps more extensive data on the scattering of polarized anti-protons on nuclei will be forthcoming from GSI.

\section{QCD Sum Rules}

QCD sum rules have been used to show that $V_{S} \approx-V_{V}$ in nuclear matter [13]. Since the derviation is too complex to repeat here, we shall only attempt to justify the result.

The vector potential will be proportional to the nuclear matter density, which in nuclear matter is uniform, $V_{V} \approx \rho_{N}$. The value of $\rho_{N}$ is taken to be the central matter density of nuclei which has been measured in electron scattering from nuclei.

In order to determine the scalar potential we need to know the nuclear scalar density. There is no direct measurement of the nuclear scalar density, but the scalar density of quarks in a nucleon can be measured experimentally. This scalar density of quarks is given in terms of what is called the sigma term

$$
\sigma_{N}=2 m_{q}(\langle N|\bar{q} q| N\rangle-\langle 0|\bar{q} q| 0\rangle)
$$

where $q$ is quark field operator, $\bar{q}=q^{\dagger} \gamma_{0}, m_{q}$ is the quark mass, $|N\rangle$ is the nucleon state, and $|0\rangle$ is the vacuum state. This sigma term can be measured in pion-nucleon scattering $[25,26]$ and, after a sophisticated analysis, is determined to be $\sigma_{N} \approx 45 \pm 8 \mathrm{MeV}$. Averaging over all the nucleons in nuclear matter and ignoring nuclear interactions,

$$
\sigma_{N} \rho_{N}=2 m_{q}\left(\langle\bar{q} q\rangle_{\rho_{N}}-\langle\bar{q} q\rangle_{\mathrm{vac}}\right)
$$

The scalar density of quarks in nuclear matter relative to the vacuum is then

$$
\frac{\langle\bar{q} q\rangle_{\rho_{N}}}{\langle\bar{q} q\rangle_{\mathrm{vac}}}=1+\frac{\sigma_{N} \rho_{N}}{2 m_{q}\langle\bar{q} q\rangle_{\mathrm{vac}}}=1-\frac{\sigma_{N} \rho_{N}}{m_{\pi}^{2} f_{\pi}^{2}}
$$

where the last term follows from the Gell-Mann-Oakes-Renner relation [27],

$$
2 m_{q}\langle\bar{q} q\rangle_{\mathrm{vac}}=-m_{\pi}^{2} f_{\pi}^{2}
$$

Both the nucleon mass and the scalar potential are Lorentz scalars. The effective mass will be proportional to the left-hand side of Equation (6) with the first term on the right-hand side proportional to the mass of the nucleon and the second proportional to the scalar potential.

The detailed QCD sum rule gives

$$
\begin{aligned}
V_{S} & =-\frac{4 \pi^{2} \sigma_{N} \rho_{N}}{M^{2} m_{q}} \\
V_{V} & =\frac{32 \pi^{2} \rho_{N}}{M^{2}}
\end{aligned}
$$

Since all the quantities on the right-hand side of Equation (8b) are positive, the scalar potential is attractive and the vector potential is repulsive, just as one finds in relativistic mean field models. Furthermore the ratio becomes

$$
\frac{V_{S}}{V_{V}}=-\frac{\sigma_{N}}{8 m_{q}} \approx-1.1
$$

using accepted values of the average quark mass in the proton $(\approx 5 \mathrm{MeV})$ and the value of $\sigma_{N}$ $(\approx 45 \mathrm{MeV})$, which is uncannily close to the the ratio of the potentials at the center of the nucleus as determined in relativistic mean field models and indicative of pseudospin symmetry. Also the negative sign originates in the vacuum expectation of the quark scalar density given in Equation (7). These features suggest that perhaps pseudospin has a more fundamental foundation in terms of QCD. 


\section{Quark Models with Spin and Pseudospin Symmetries}

A successful phenomenological model of quarks uses the non-relativistic Schrodinger Hamiltonian with the Cornell potential

$$
V(r)=-\frac{\alpha_{s}}{r}+\sigma r
$$

The first term arises from the single gluon exchange between quarks while the second term leads to confinement of the quarks inside the hadrons. Recently this Cornell potential was used in the Dirac Hamiltonian and studied under the conditions of spin symmetry and pseudospin symmetry [28]. When spin symmetry was imposed the effective energy-dependent potential derived in the non-relativistic limit was a Cornell type potential which is consistent with hadron phenomenology. However when pseudospin symmetry was imposed the effective energy-dependent potential derived in the non-relativistic limit was quite different and was bag-like. The size of the bag was comparable to the size of the nucleus. This is unrealistic because quarks in nuclei are confined to the size of the nucleon and do not traverse the size of the nucleus as a whole.

However, this is an interesting line of research which should be explored more. In particular the question of why hadrons have spin symmetry while nuclei have pseudospin symmetry needs to be answered.

\section{Conclusions}

Most features of nuclei can be understood within the non-relativistic shell model. Therefore, it comes as a surprise that pseudospin symmetry in nuclei is a relativistic symmetry. However, it can never be an invariant symmetry since no nucleon bound states exist when the sum of the scalar and vector potentials is a constant.

Approximate pseudospin symmetry in nuclei implies that spin symmetry will be approximately conserved in anti-nucleon scattering from nuclei. The new anti-proton facility at GSI could test this prediction by measuring the polarization of anti-protons scattered from nuclei. This prediction follows simply from charge conjugation and, if confirmed, will be another manifestation of the relativistic origin of pseudospin symmetry.

In addition, since QCD sum rules predict that the sum of the scalar and vector potentials is small in nuclear matter, perhaps there is a more fundamental rationale for pseudospin symmetry in terms of quark dynamics. With this understanding may come an answer to the question of why hadrons exhibit spin symmetry whereas nuclei exhibit pseudospin symmetry.

Acknowledgments: This research was supported by the US Department of Energy, Contract No. W-7405-ENG-36.

Conflicts of Interest: The author declares no conflict of interest.

\section{References}

1. Arima, A.; Harvey, M.; Shimizu, K. The empirical p-n interactions and atomic masses. Phys. Lett. B 1969, 30 , doi:10.1088/1742-6596/403/1/012045.

2. Hecht, K.T.; Adler, A. Generalized seniority for favored $\mathrm{J} \neq 0$ pairs in mixed configurations. Nucl. Phys. A 1969, 137, 129-143.

3. Sorlin, O.; Porquet, M.G. Nuclear magic numbers: new features far from stability. Prog. Part. Nucl. Phys. 2008, 61, 602-673.

4. Bohr, A.; Hamamoto, I.; Mottelson, B.R. Pseudospin in rotating nuclear potentials. Phys. Scr. 1982, 26, doi:10.1088/0031-8949/26/4/003.

5. Ginocchio, J.N. Pseudospin as a relativistic symmetry. Phys. Rev. Lett. 1997, 78, doi:10.1103/PhysRevLett.78.436.

6. Ginocchio, J.N. Relativistic symmetries in nuclei and hadrons. Phys. Rep. 2005, 414, 165-261. 
7. Liang, H.; Meng, J.; Zhou, S.G. Hidden pseudospin and spin symmetries and their origins in atomic nuclei. Phys. Rep. 2015, 570, 1-84.

8. Bell, J.S.; Ruegg, H. Dirac equations with an exact higher symmetry. Nucl. Phys. B 1975, 98, 151-153.

9. Page, P.R.; Goldman, T.; Ginocchio, J.N. Relativistic symmetry Suppresses quark spin-orbit splitting. Phys. Rev. Lett. 2001, 86, doi:10.1103/PhysRevLett.86.204.

10. Ginocchio, J.N. A relativistic symmetry in nuclei. Phys. Rep. 1999, 315, 231-240.

11. Ginocchio, J.N.; Leviatan, A. On the relativistic foundations of pseudospin symmetry in nuclei. Phys. Lett. B 1998, 425, 1-5.

12. Blokhin, A.L.; Bahri, C.; Draayer, J.P. Origin of pseudospin symmetry. Phys. Rev. Lett. 1995, 74, doi:10.1103/PhysRevLett.74.4149.

13. Cohen, T.D.; Furnstahl, R.J.; Griegel, D.K.; Jin, X. QCD sum rules and applications to nuclear physics. Prog. Part. Nucl. Phys. 1995, 35, 221-298.

14. Ginocchio, J.N.; Madland, D.G. Pseudospin symmetry and relativistic single-nucleon wave functions. Phys. Rev. C 1998, 57, doi:10.1103/PhysRevC.57.1167.

15. Meng, J.; Sugawara-Tanabe, K.; Yamaji, S.; Ring, P.; Arima, A. Pseudospin symmetry in relativistic mean field theory. Phys. Rev. C 1998, 58, doi:10.1103/PhysRevC.58.R628.

16. Sugawara-Tanabe, K.; Yamaji, S.; Arima, A. Spin symmetry and pseudospin symmetry in the relativistic mean field with a deformed potential. Phys. Rev. C 2002, 65, doi:10.1103/PhysRevC.65.054313.

17. Ginocchio, J.N.; Leviatan, A.; Meng, J.; Zhou, S.G. Test of pseudospin symmetry in deformed nuclei. Phys. Rev. C 2004, 69, doi:10.1103/PhysRevC.69.034303.

18. Ginocchio, J.N. Pseudospin symmetry and relativistic mean field eigenfunctions. Phys. Rev. C 2002, 66, doi:10.1103/PhysRevC.66.064312.

19. Ginocchio, J.N. Implications of pseudospin symmetry on relativistic magnetic properties and Gamow-Teller transitions in nuclei. Phys. Rev. C 1999, 59, doi:10.1103/PhysRevC.59.2487.

20. Von Neumann-Cosel, P.; Ginocchio, J.N. l-forbidden M1 transitions and pseudospin symmetry. Phys. Rev. C 2000, 62, doi:10.1103/PhysRevC.62.014308.

21. Hagberg, E.; Alexander, T.K.; Neeson, I.; Koslowsky, V.T.; Ball, G.C.; Dyck, G.R.; Forster, J.S.; Hardy, J.C.; Leslie, J.R.; Mak, H.B.; et al. Confirmation of the $l$-forbidden Gamow-Teller decay branch of ${ }^{39}$ Ca. Nucl. Phys. A 1994, 571, 555.

22. Zhou, S.G.; Meng, J.; Ring, P. Spin symmetry in the antinucleon spectrum. Phys. Rev. Lett. 2003, 91, doi:10.1103/PhysRevLett.91.262501.

23. Ginocchio, J.N. U(3) and pseudo-U(3) symmetry of the relativistic harmonic oscillator. Phys. Rev. Lett. 2005, 95, doi:10.1103/PhysRevLett.95.252501.

24. Martin, A.; Birsa, R.; Bos, K.; Bradamante, F.; Bugg, D.V.; Torre-Colautti, S.D.; Hall, J.R.; Heer, E.; Hess, R.; Kluyver, J.C.; et al. Polarization at small angles in antiproton-carbon elastic scattering at lear energies. Nucl. Phys. A 1988, 487, 563-590.

25. Gasser, J.; Leutwyler, H.; Saino, M.E. Sigma-term update. Phys. Lett. B 1991, 253, 252-259.

26. Saino, M.E. Pion-nucleon sigma-term-A review. In Proceedings of the Ninth International Symposium on Meson-Nucleon Physics and the Structure of the Nucleon, Washington, DC, USA, 26-31 July 2001.

27. Gell-Mann, M.; Oakes R.J.; Renner, B. Behavior of Current Divergences under SU3 × SU3. Phys. Rev. 1968, 175, doi:10.1103/PhysRev.175.2195.

28. Kosyakov, B.P.; Popov, E.Y.; Vronskii, M.A. The bag and the string: Are they opposed? Phys. Lett. B 2015, 77, $28-33$.

(C) 2016 by the author; licensee MDPI, Basel, Switzerland. This article is an open access article distributed under the terms and conditions of the Creative Commons by Attribution (CC-BY) license (http://creativecommons.org/licenses/by/4.0/). 